\title{
Application of fast CCD drift scanning to speckle imaging of binary stars
}

\author{
O. Fors ${ }^{1,2}$, E. P. Horch ${ }^{3}$, and J. Núñez ${ }^{1,2}$ \\ ${ }^{1}$ Departament d'Astronomia i Meteorologia, Universitat de Barcelona, Av. Diagonal 647, 08028 Barcelona, Spain \\ 2 Observatori Fabra, Camí de l'Observatori s/n, 08035 Barcelona, Spain \\ 3 Department of Physics, University of Massachusetts Dartmouth, 285 Old Westport Road, North Dartmouth, MA, USA
}

Received 24 July 2003/ Accepted 24 February 2004

\begin{abstract}
A new application of a fast CCD drift scanning technique that allows us to perform speckle imaging of binary stars is presented. For each observation, an arbitrary number of speckle frames is periodically stored on a computer disk, each with an appropriate exposure time given both atmospheric and instrumental considerations. The CCD charge is shifted towards the serial register and read out sufficiently rapidly to avoid an excessive amount of interframe dead time. Four well-known binary systems (ADS 755, ADS 2616, ADS 3711 and ADS 16836) are observed in to show the feasibility of the proposed technique. Bispectral data analysis and power spectrum fitting is carried out for each observation, yielding relative astrometry and photometry. A new approach for self-calibrating this analysis is also presented and validated.

The proposed scheme does not require any additional electronic or optical hardware, so it should allow most small professional observatories and advanced amateurs to enjoy the benefits of diffraction-limited imaging.
\end{abstract}

Key words. instrumentation: detectors - binaries: visual - techniques: interferometric techniques: high angular resolution - astrometry

\section{Introduction}

Over the last few years, CCDs have been used with increasing frequency for speckle imaging. This work actually started more than a decade ago with the work of Beletic \& Zadnik (1993), and has more recently been extended by Horch et al. (1997, 1999) and Kluckers et al. (1997).

There are three main reasons for this change. First, CCDs have dramatically improved in terms of their readout noise and speed characteristics. Second, it has been realized that changing the readout pattern allows one to use large-format CCDs effectively in speckle imaging. Finally, there has been the hope that CCDs would allow diffraction-limited photometry in a way that intensified cameras, such as ICCDs and other microchannel-plate-based devices have not been able to do up to the present.

In this paper, we show that CCD-based speckle imaging can be extended to a relatively inexpensive detection system, namely the Santa Barbara Instruments Group (SBIG) ST-8 camera, which in particular does not have the speed and readout flexibility that other CCDs being used for the same purpose have.

Fast drift scanning has already been shown to be a useful technique for recording lunar occultations at millisecond sampling rate (Fors et al. 2001, 2003).

Send offprint requests to: O. Fors, e-mail: ofors@am.ub.es
In the case of speckle observations, a fast drift scanning acquisition scheme is adapted to meet the specific needs of speckle imaging. The data are read out off the chip as fast as possible, but with pauses in readout corresponding to the collection of speckle patterns. In addition, we introduce a method of self-calibration of speckle data in the Fourier plane which makes possible to make reconstructed images without taking data on an unresolved point source. Finally, we make some recommendations which may be valuable for small observatories or advanced amateurs should they wish to carry this work forward.

\section{Proposed technique basis}

Fors et al. (2001, 2003) showed that fast drift scanning can be applied to obtain high-resolution measurements by means of lunar occultation (LO) observations. In that approach, the occultation lightcurve was recorded by reading out every millisecond the small fragment of the CCD in which the object was situated. This procedure was continuously maintained until the occultation event took place.

In this paper we present a variation of the former acquisition technique. As in the LO approach, the telescope tracking is turned on and the shutter remains open throughout the observation. To perform speckle imaging, the continuous column readout is periodically interrupted by an amount of time which 


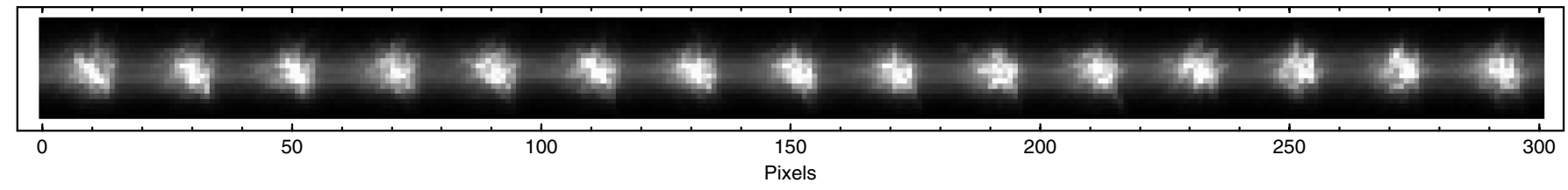

Fig. 1. Raw strip image of ADS 755 as observed when following the proposed technique. Specklegrams are $20 \times 20$ pixels in size and the exposure time is $39 \mathrm{~ms}$.

is the effective speckle frame exposure time. The resulting image of this process is an arbitrarily long strip with a series of speckle frames.

Of course, the camera spends some measurable time reading out all columns of each speckle frame. As a result of that unavoidable dead time between consecutive speckle frames, a low-level streaking appears between speckle images. In general, the importance of this effect will depend on the camera specifications, namely digitization and data transfer rate.

The proposed acquisition scheme is directly applicable to any full frame CCD camera for which it is possible to set readout column rate and size by software means. No hardware or optical modification has to be made to the telescope to make this technique possible.

Large format CCDs had already been used for speckle imaging in the past by one of us (Horch et al. 1997). In that approach, called fast subarray readout, ten to twenty speckle frames were stored in a subarray strip of the KAF-4200 chip until it became filled. Afterwards, the shutter was closed and the whole subarray was readout. The proposed technique in the current article exhibits one advantage and one disadvantage with respect to fast subarray readout. On the one hand, one can now obtain as many speckle frames as desired without periodically closing the shutter: it is not limited by CCD chip size as in the subarray-readout mode. On the other hand, it is necessary to read out all the columns of the CCD between consecutive speckle frame exposures. This yields a longer dead time, which increases low-level streaking. However, it is likely the dead time will be significantly reduced in the very near future with new faster CCD cameras available on the professional and high-end amateur market (see Sect. 6.1 for further discussion on this topic).

The term fast drift scanning for both speckle imaging and lunar occultations may seem somewhat ambiguous. In a strict sense, term should only be used when the RA tracking drive is turned off and, as a result, the imaged scene drifts over the CCD chip at the same rate the column charge is clocked towards the serial register. However, to be consistent with Fors et al. (2001) we will adopt the same designation.

\section{Observations}

Speckle observations were conducted at the $1.5 \mathrm{~m}$ telescope of the Observatorio Astronómico Nacional at Calar Alto (Spain) in October, 2001. The same camera employed by LO at Fors et al. $(2001,2003)$ was used. This is a Texas Instruments TC-211 CCD, set inside an SBIG ST8 camera as the tracking chip. It is a full frame front-illuminated CCD with $13.75 \times 16 \mu \mathrm{m}$ pixels and a $192 \times 164$ pixel format. It is read out through a parallel port interface and its electronic module can be operated at $30 \mathrm{kHz}$ with 12 electrons rms readout noise. The camera was directly attached at the Cassegrain focus of the telescope without any magnification optics. This configuration yields an effective focal length of $12280 \mathrm{~mm}$.

Four binary systems were observed during 5 nights (see Cols. 1-6 in Table 1 for details), under median seeing conditions of $1.3^{\prime \prime}$. Those objects were selected because they have well determined orbits which allow us to validate the acquisition technique described in Sect. 2. Several speckle frame sequences were obtained for every object. Each sequence consists of a few hundreds of frames.

All speckle observations were conducted with a Cousins $R$ filter $(\lambda=641 \pm 100 \mathrm{~nm})$. At this wavelength, the diffraction-limited spot size is equal to 108 mas. On the other hand, the scale calibration was carried out by means of a standard plate solution of long exposure frames, and was found to be $9.375 \mathrm{mas} \mathrm{mm}^{-1}$. Thus, our data is undersampled and this will be taken into account in the reduction process (see Sect. 4).

Data acquisition was performed using an implementation of the proposed technique into a DOS-based program called $\mathrm{SCAN}^{1}$. This program offers satisfactory relative timing accuracy when scheduling column readout at millisecond rates.

In CCD-based speckle imaging there is a competition between readout noise and atmospheric correlation time. On the one hand, longer frame integration times give you more photons, which gives better contrast of the speckle pattern with the readout noise. On the other hand, you lose speckle contrast if too long frame time is used. Therefore, it is not just an instrumental readout limitation that forces us to use a frame time longer than the correlation time, but it is desirable to minimize the effect of CCD read noise.

In Fig. 1 we show a subset of a typical sequence of speckle frames obtained by means of this technique. For this particular case, a 20-pixel column is stored every $1.8 \mathrm{~ms}$ on average, yielding a dead time of $36 \mathrm{~ms}$. This must be added to the exposure time, $39 \mathrm{~ms}$. Note that this is significantly larger than the typical atmospheric coherence time for seeing of $1.3 \mathrm{arcsec}$, which has been estimated at several observatories to be on the order of 4-8 ms. The choice of this longer exposure time and its consequences for data quality is justified and discussed in Sect. 4.

\footnotetext{
1 Available at http://www.driftscan.com
} 


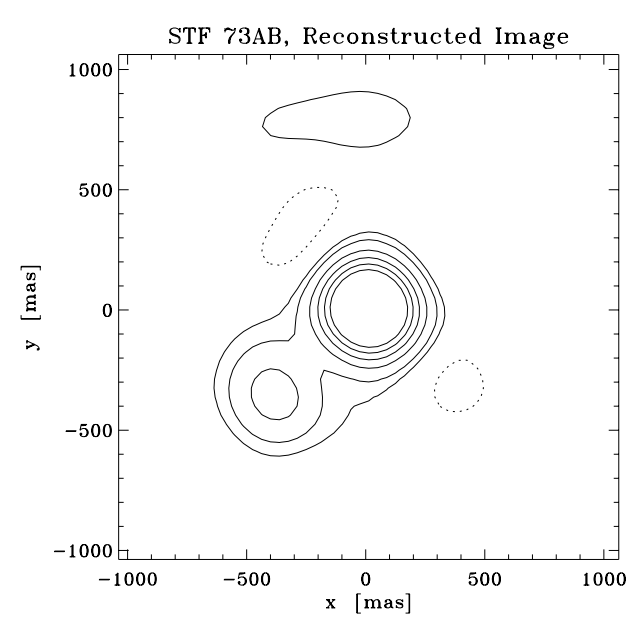

Fig. 2. A reconstructed image of WDS $00550+2338=\operatorname{ADS} 755=$ STF 73AB. North is down, East is to the right. Contours are drawn at $-0.05,0.05,0.10,0.20,0.30,0.40$, and 0.50 of the maximum value in the array. The dotted contours indicate the value -0.05 . The secondary star appears below and to the left of the primary, which is located in the center of the image. The feature in the upper part of the figure is not real and appears to be related to the mismatch between the seeing profile of the binary observation and the radially generated point source.

\section{Data analysis and self-calibration}

Once the raw data are read out of the camera, pixels around the object of interest are extracted and converted to FITS format. The FITS file is stored as an image stack where each image contains a $20 \times 20$ pixel speckle pattern. Approximately 500 of such images are contained in the stack of a single observation. These files are then analyzed in exactly the same way as described in Horch et al. (1997). Briefly, the method is to subtract the bias level and the streak between images caused by the readout scheme, and then to compute the autocorrelation and low-order bispectral subplanes needed for subsequent analysis.

In the case of reconstructed images, the relaxation technique of Meng et al. (1990) is used to generate a phase map of the object's Fourier transform, and this is combined with the object's modulus obtained by taking the square root of the power spectrum. By combining the modulus and the phase and inverse transforming, one arrives at the reconstructed image. An example of such an image is shown in Fig. 2.

In the case of deriving relative astrometry of binary stars, the weighted least squares approach of Horch et al. (1996) has been used. This method fits a power spectrum deconvolved by a point source calibrator to a trial fringe pattern and then attempts to minimize the reduced chi-squared of the function. The undersampling correction of Horch et al. (1997) is applied.

For all data discussed here, an estimate for an unresolved point source power spectrum was constructed from that of a binary star. This has the advantage of allowing binary star observations to be taken without interrupting for measurements of the speckle transfer function. A synthetic point source estimate can be generated first by forming the power spectrum of any binary (see Fig. 3), and then extracting a trace from the

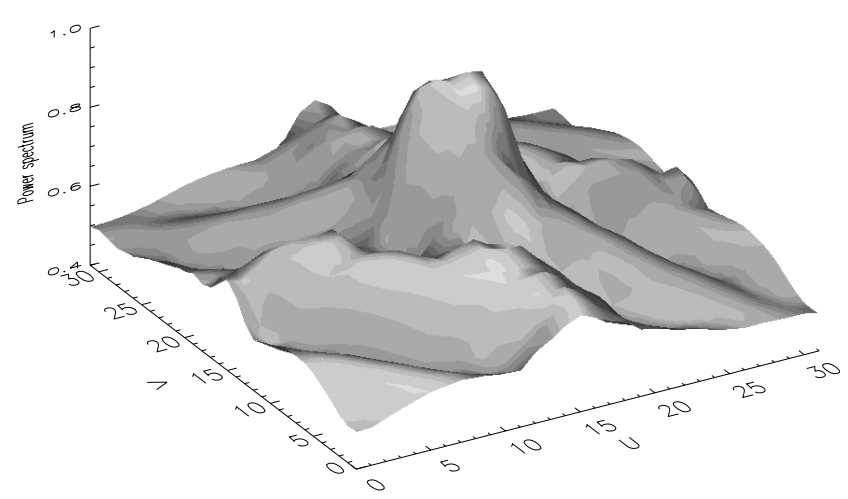

Fig. 3. A surface plot of the power spectrum of one of the observing runs for ADS 755. Note the fringe pattern due to binarity of the object.

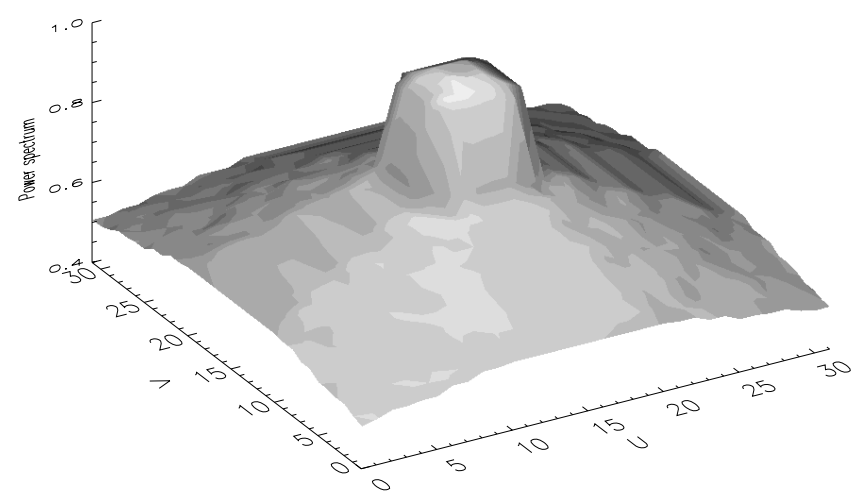

Fig. 4. Power spectrum for a calculated point source following the self-calibration scheme. Compared to Fig. 3, the central peak due to seeing remains approximately the same and fringes in the speckle shoulder are not present, as expected.

image along the central fringe. Since the binary is not resolved along this direction, this is essentially a one-dimensional estimate of an unresolved source. This one-dimensional function is then rotated about the origin of the frequency plane to fill a two-dimensional array. This generates a radially symmetric function, as indeed a true unresolved source should show under perfect conditions (see Fig. 4). The method has limitations as we will discuss in Sect. 6.2 after the main body of results has been presented, but provides a way to make the deconvolution needed without recourse to point source observations.

As noted in Sect. 3, the speckle frame exposure time was chosen to be larger than the coherence time. This choice is justified by the competition between readout noise and correlation time when performing CCD-based speckle imaging. On the one hand, speckle frames show the highest possible signalto-noise ratio when the integration time is in fact longer than the coherence time. One of us (E.H.) has shown that $50 \mathrm{~ms}$ is the exposure time where the maximum in the signal-to-noise ratio occurs at the WIYN telescope, which uses a CCD with a readout noise of 10 electrons. That probably implies a factor of 4 to 5 larger than the coherence time (Horch et al. 2002). On the other hand, in general speckle contrast decreases as longer exposure time are used. Therefore, it is not just an instrumental readout limitation that forces us to use a frame time longer than the correlation time, but it is desirable to minimize the 
Table 1. Double star speckle measures.

\begin{tabular}{|c|c|c|c|c|c|c|c|c|}
\hline ADS & $\begin{array}{l}\text { Discoverer } \\
\text { Designation }\end{array}$ & HD & HIP & $\begin{array}{c}\text { WDS } \\
(\alpha, \delta \mathrm{J} 2000.0)\end{array}$ & $\begin{array}{l}\text { Date } \\
\text { (BY) }\end{array}$ & $\begin{array}{r}\theta \\
\left({ }^{\circ}\right)\end{array}$ & $\begin{array}{r}\rho \\
\left({ }^{\prime \prime}\right)\end{array}$ & $\Delta m$ \\
\hline \multirow[t]{3}{*}{755} & STF 73AB & 5286 & 4288 & $00550+2338$ & 2001.8127 & 311.5 & 0.935 & $1.17^{b}$ \\
\hline & & & & & 2001.8178 & 310.5 & 0.936 & 0.43 \\
\hline & & & & & 2001.8207 & 311.0 & 0.936 & 0.53 \\
\hline \multirow[t]{2}{*}{2616} & STF $412 \mathrm{AB}$ & 22091 & 16664 & $03344+2428$ & 2001.8208 & 175.8 & 0.651 & $0.35^{a}$ \\
\hline & & & & & 2001.8261 & 176.2 & 0.646 & $0.58^{a}$ \\
\hline 3711 & STT 98 & 33054 & 23879 & $05079+0830$ & 2001.8157 & 319.8 & 0.743 & 0.52 \\
\hline \multirow[t]{3}{*}{16836} & BU 720 & 221673 & 116310 & $23340+3120$ & 2001.8124 & 95.8 & 0.560 & $0.57^{b}$ \\
\hline & & & & & 2001.8208 & 97.2 & 0.585 & 0.36 \\
\hline & & & & & 2001.8260 & 92.8 & 0.589 & $0.90^{b}$ \\
\hline
\end{tabular}

${ }^{a}$ There is an ambiguity of 180 degrees in the position angle compared to previous observations.

${ }^{b}$ Observation was taken at low elevation. This may affect the quality of the result.

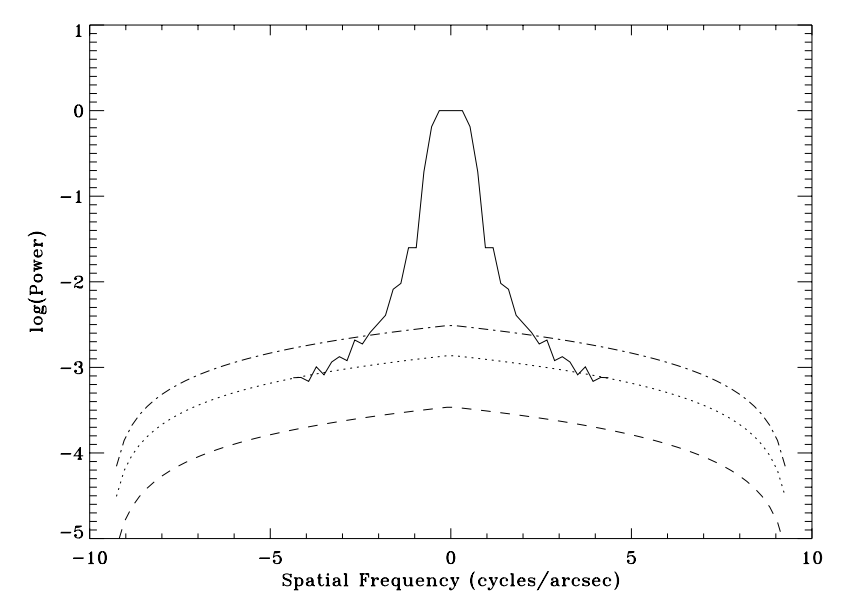

Fig. 5. Comparison of cutoff frequencies of observed and simulated 1-D speckle transfer functions. The former (solid line) was generated from the ADS 2616 point source. The latter represents the diffraction limited power spectrum obtained at $641 \mathrm{~nm}$ using a $1.5 \mathrm{~m}$ aperture. Three different values of the Fried parameter $r_{0}, 5 \mathrm{~cm}$ (dashed), $10 \mathrm{~cm}$ (dotted), and $15 \mathrm{~cm}$ (dash-dotted), have been considered. Note that the better the seeing, the larger $r_{0}$ and so the higher the curve on the plot.

effect of CCD read noise, while still preserving sufficient contrast on speckle patterns.

In addition, interframe dead time contributes to low-level streaking. However, note that the light contributing to streaking is distributed far more uniformly and over more pixels than those forming the speckle pattern itself. As a result, the ratio between intensity peaks is much more favorable than the ratio between dead time and atmospheric coherence time.

All this introduces attenuation in the higher frequencies of our data. To illustrate how this affects resolution, a plot with four 1D power spectrum curves has been made. As shown in Fig. 5, one corresponds to an observed point source and the other three to the diffraction limited spot one would obtain with the instrumental conditions of current paper. The attenuation factor used for generating such simulated profiles is given by:

$A=0.435\left(r_{0} / D\right)^{2}$ where $r_{0}$ is the Fried parameter and $D$ the telescope diameter. The 0.435 is a geometrical factor derived by Korff (1973) and Fried (1979).

Ideally, the high-frequency portion of the speckle transfer function should overlap to the simulated curve attenuated with the $r_{0}$ value which best matches the real seeing. However, due to the significant undersampling of our data, the observed power spectrum does not span up to the theoretical diffraction

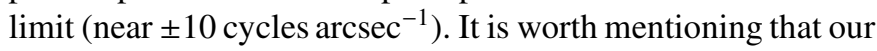
reduction software does account for the aliasing effect of the undersampling and, in principle, is able to extract part of those frequencies which are aliased to lower frequencies. However, this last is somewhat limited by the low signal-to-noise which these high frequencies show. Thus, we see that the impact of longer exposure time is relatively small, and does not handicap our data quality.

\section{Results}

In Table 1 we show all speckle measures obtained during our five night observing run after applying the self-calibration analysis as explained in the previous section. Column headings are as follows: (1) the Aitken Double Star number; (2) the discoverer designation as it appears in the Washington Double Star Catalog (WDS); (3) the Henry Draper Catalogue number; (4) the HIPPARCOS Catalogue number; (5) the Washington Double Star Catalogue number, which is the same as the position in 2000.0 coordinates; (6) the date in fraction of the Besselian year when the observation was made; (7) the position angle $(\theta)$ with north through east defining the positive sense of the angle; (8) the separation $(\rho)$ in arc seconds; and (9) the magnitude difference as judged from the speckle observations. Position angles are not corrected for precession and are therefore valid for the epoch of observation shown. Every $\left(\rho, \theta, \Delta_{m}\right)$ triplet in the table is the result of averaging the result of 5 frame sequences, which were exposed within a few minutes of each other. As indicated in the table, some observations were taken at low elevation. Note that position angle, separation, and magnitude differences for these measures 


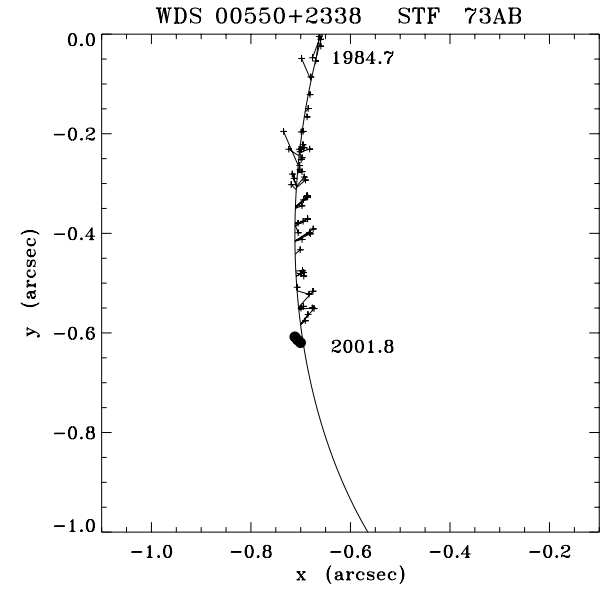

(a)

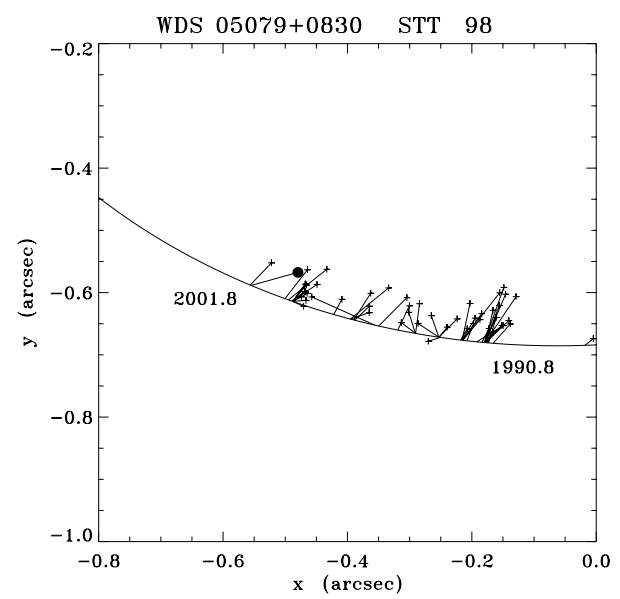

(c)

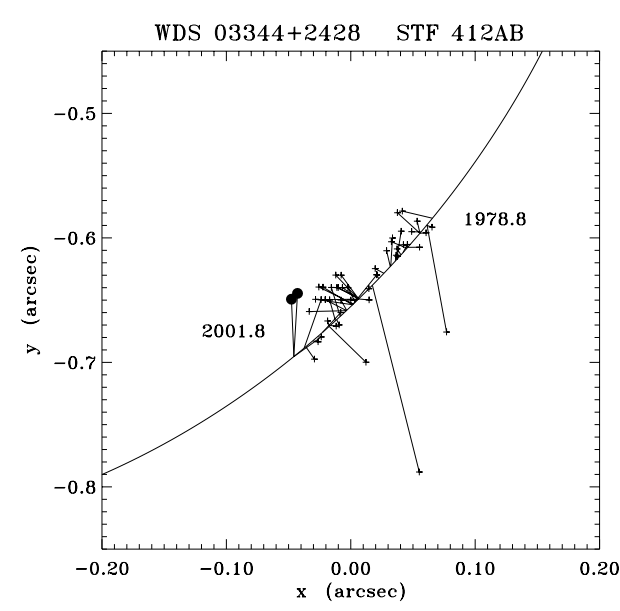

(b)

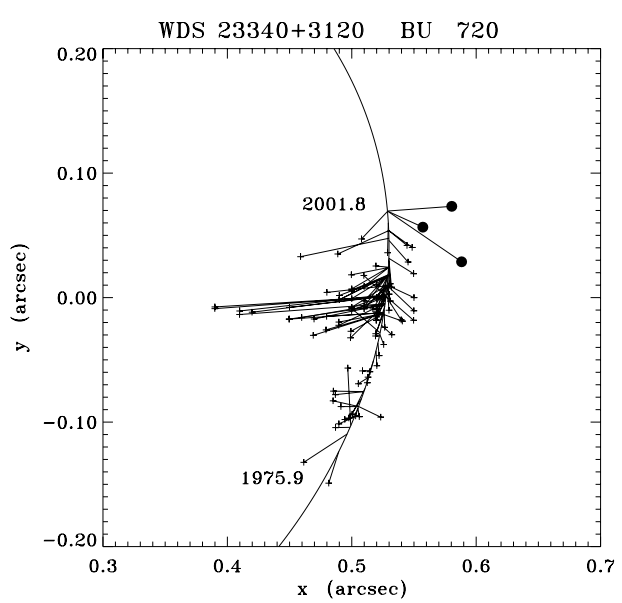

(d)

Fig. 6. A comparison of the position angle and separation measures presented here with the work of other observers. In all plots, North is down and East is to the right. In all cases, the object has an orbit listed in the 6th Catalog of Orbits of Visual Binary Stars (Hartkopf et al. 2003), and the orbital trajectory is plotted. Observations of previous observers, compiled by Hartkopf et al. (2002), are marked with small plus symbols, with a line segment drawn from the point to the ephemeris prediction for that epoch. The observations presented here are marked with the solid dots, again with line segments joining the point to the predicted location given the orbital elements. a) WDS $00550+2338=\mathrm{STF} 73 \mathrm{AB}=\mathrm{ADS} 755$. The orbit plotted is that of Docobo \& Costa (1990), rated as a Grade 2 orbit in the Sixth Catalog. b) WDS 03344+2428 = STF 412AB = ADS 2616. The orbit plotted is that of Scardia et al. (2002), rated as a Grade 3 orbit in the Sixth Catalog. c) WDS $05079+0830=$ STT $98=$ ADS 3711. The orbit plotted is that of Baize (1969), rated as a Grade 3 orbit in the Sixth Catalog. d) WDS 23340+3120= BU $720=$ ADS 16836 . The orbit plotted is that of Starikova (1982), rated as a Grade 3 orbit in the Sixth Catalog.

appear discrepant from the rest of values. Therefore, the selfcalibration point source method should be used only at modest zenith angles (less than thirty degrees, if no atmospheric dispersion compensation is performed). Further discussion about this limitation will be given in Sect. 6.2.

In Fig. 6 we compare the obtained results with those from other observers and the predicted orbit for each object. In general, our measure orbit offsets are within the global scatter of all other positions. Those that are farthest from the orbital ephemeris positions correspond, again, to observations performed at low elevation. The point source calibrator in all cases was generated from a high signal-to-noise observation of ADS 755.

Assuming no major systematic errors, the total uncertainty for the measures in Table 1 can be estimated by combining the uncertainty generated from night-to-night scatter when using the same point source and the variation in the result obtained by using different point source calibrators. Errors resulting from the fitting procedure are not considered since they have been found to be an small fraction $(\sim 5 \%)$ of the overall uncertainty of a given measure of $\rho, \theta$ and $\Delta_{m}$. Although the data set here does not permit definitive uncertainty estimates due to the small sample of objects observed, we can nonetheless make first order estimates of these quantities. Firstly, we obtain the night-to-night scatter $\left(\sigma^{\mathrm{nn}}\right)$ by computing the standard deviations of the two objects in Table 1 with the largest number of observations, and averaging those two quantities. Secondly, we estimate point source error $\left(\sigma^{\mathrm{ps}}\right)$ by making use of the values in Table 2, which are also displayed in Fig. 7. This table includes $\left(\rho, \theta, \Delta_{m}\right)$ results obtained when using different point source calibrators for one single speckle sequence. The average of the two rows designated as $\sigma$ represents an 
Table 2. Comparison of results obtained with different point source power spectra.

\begin{tabular}{|c|c|c|c|c|c|c|c|c|}
\hline ADS & $\begin{array}{l}\text { Discoverer } \\
\text { Designation }\end{array}$ & $\mathrm{HD}$ & HIP & $\begin{array}{c}\text { WDS } \\
(\alpha, \delta \mathrm{J} 2000.0)\end{array}$ & $\begin{array}{l}\text { Date } \\
\text { (BY) }\end{array}$ & $\begin{array}{r}\theta \\
\left({ }^{\circ}\right) \\
\end{array}$ & $\begin{array}{r}\rho \\
\left({ }^{\prime \prime}\right) \\
\end{array}$ & $\Delta m$ \\
\hline \multirow[t]{5}{*}{755} & STF 73AB & 5286 & 4288 & $00550+2338$ & 2001.8207 & 311.7 & 0.939 & 0.76 \\
\hline & & & & & 2001.8207 & 312.5 & 0.945 & 0.98 \\
\hline & & & & & 2001.8207 & 311.7 & 0.936 & 0.73 \\
\hline & & & & & 2001.8207 & 311.1 & 0.938 & 0.59 \\
\hline & & & & $\sigma$ & & 0.6 & 0.004 & 0.16 \\
\hline \multirow[t]{6}{*}{2616} & STF $412 A B$ & 22091 & 16664 & $03344+2428$ & 2001.8261 & 178.4 & 0.674 & 0.58 \\
\hline & & & & & 2001.8261 & 174.5 & 0.642 & 0.31 \\
\hline & & & & & 2001.8261 & 176.2 & 0.664 & 0.23 \\
\hline & & & & & 2001.8261 & 173.5 & 0.634 & 0.35 \\
\hline & & & & & 2001.8261 & 178.4 & 0.672 & 0.70 \\
\hline & & & & $\sigma$ & & 2.2 & 0.018 & 0.20 \\
\hline
\end{tabular}

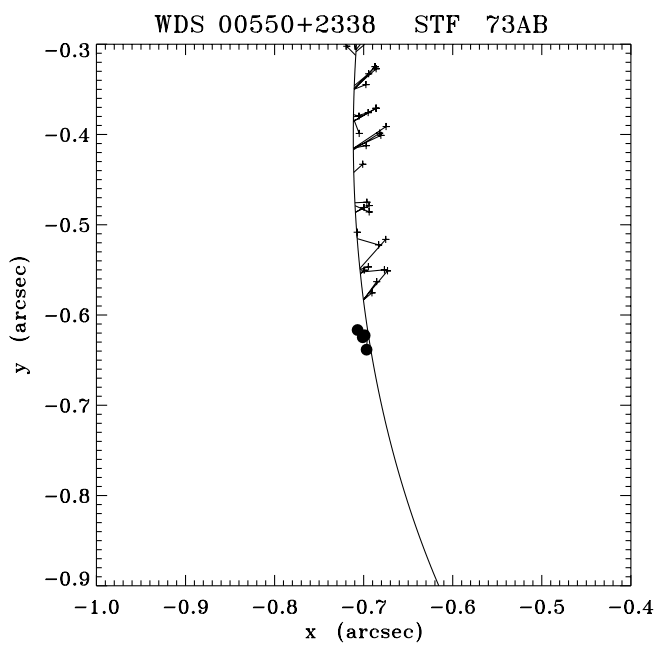

(a)

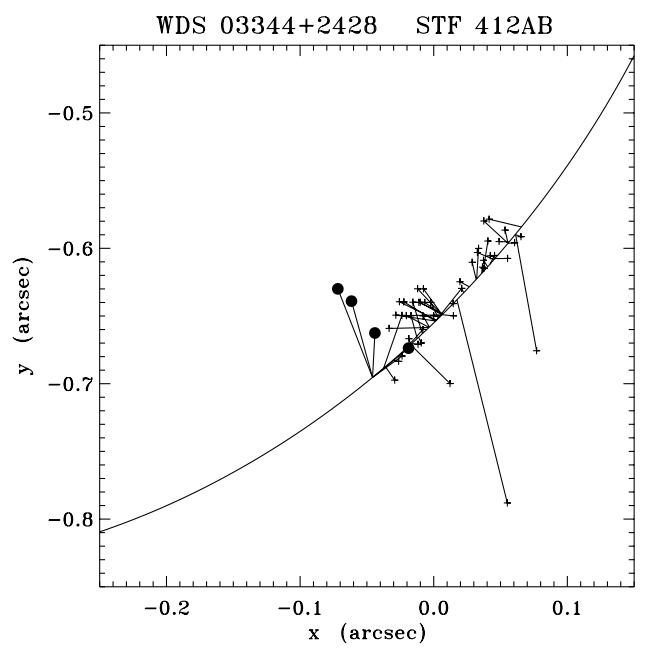

(b)

Fig. 7. Comparison of astrometric results using different point source calibrations. Point sources generated from observations of ADS 3711 and ADS 755 were used in both cases. The plot symbols and orbital trajectories are the same as in Fig. 6 . a) WDS $00550+2338=\mathrm{STF} 73 \mathrm{AB}=$ ADS 755. b) WDS 03344+2428 = STF 412AB = ADS 2616

estimate of the point source error for one observation $\left(\sigma_{1}^{\mathrm{ps}}\right)$. Whereas, $\left(\rho, \theta, \Delta_{m}\right)$ derived in Table 1 proceed from 5 consecutive speckle pattern sequences. As a result, to obtain a $\sigma^{\mathrm{ps}}$ fully comparable with $\sigma^{\mathrm{nn}}, \sigma_{1}^{\mathrm{ps}}$ has been divided by $\sqrt{n-1}, n=5$.

Finally, assuming statistical independence, we obtain the following expected uncertainties in each coordinate by adding $\sigma^{\mathrm{ps}}$ in quadrature with $\sigma^{\mathrm{nn}}$ :

Position angle: $\sigma_{\theta}=1.5^{\circ}$;

Separation: $\sigma_{\rho}=0.017^{\prime \prime}$;

Magnitude difference: $\sigma_{\Delta m}=0.34 \mathrm{mag}$.

The separation number is very similar to the result in Douglass et al. (1999) (US Naval Observatory obtained speckle results with $\sigma_{\rho}=0.018^{\prime \prime}$ using 66-cm telescope). However, $\sigma_{\theta}$ is higher in our case (Douglass et al. obtained $0.57^{\circ}$ for a $1^{\prime \prime}$ separation, although $1^{\circ}$ is a typical uncertainty in well-calibrated speckle work). $\sigma_{\Delta m}$ is probably large because of the small window used and self-calibration technique limitations.

As stated above, the point source from ADS 755 was used for the analysis of all objects. To find the degree of validity of this assumption, and to determine how significant the change in atmospheric conditions is, we have divided the point source 1D power spectrum of ADS 755 by those from ADS 16836 and ADS 755, obtained on different nights. Ideally, the resulting curves should be constant and equal to unity for all frequencies. As shown in Fig. 8, the curves appear to be quite flat over the whole frequency domain. Only marginal residuals in the range of seeing wings are visible for the two upper plots. Those are due to region of the seeing peak not being considered when the power spectrum fits are performed. The information in Fig. 8 is complementary to what is shown in Table 2. 


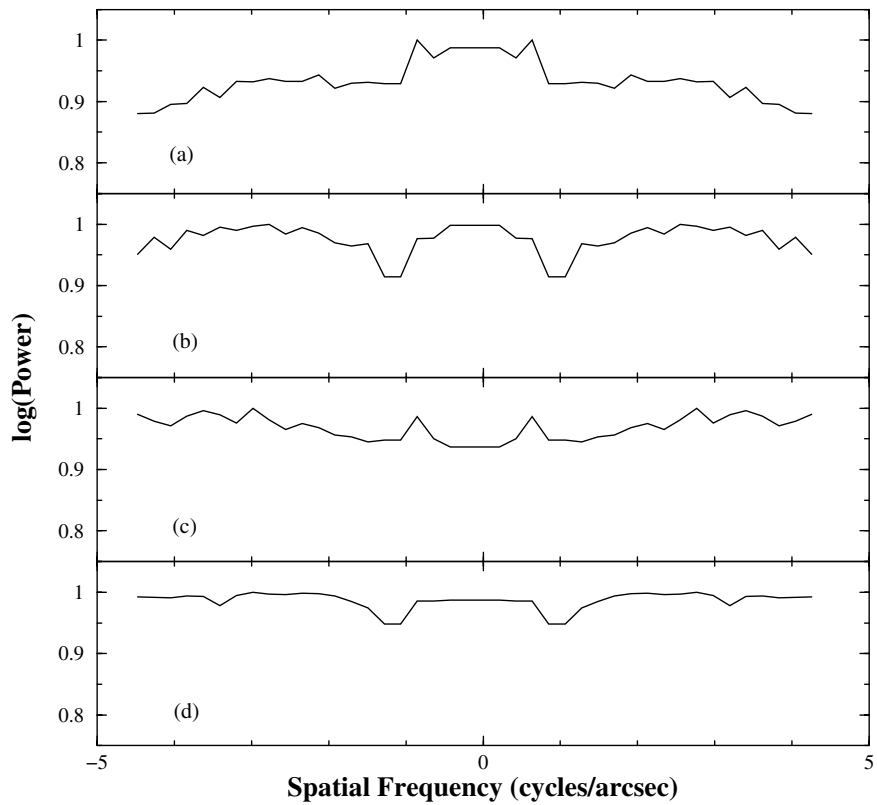

Fig. 8. Comparison of 1D point source power spectrum of ADS 755 on the 5th night of observation with respect to: a) ADS 16386 on 5th night; b) ADS 16386 on 6th night; c) ADS 755 on 2nd night; and d) ADS 755 on 4th night.

\section{Discussion}

\subsection{New CCDs improvements}

The performance of the fast drift scanning technique depends on camera readout rate, which directly fixes the minimum dead time attainable. If this is excessive, streaking could handicap the scientific usefulness of the data. In general, the readout rate of a camera will depend on its digitization level and data transfer rate. The digitization rate is fixed by CCD microcontroller design and digitization depth. The data transfer rate is specified by the port architecture being used between the camera and computer.

In the case of the camera used in current paper, its readout rate of $30 \mathrm{kpix} \mathrm{s}^{-1}$ can be considered as moderately low compared with CCDs currently in the market. The fact that it is controlled through parallel port interface fairly limits the final readout rate. This is not surprising, since SBIG-ST8 was designed for general CCD stare imaging purposes, where a long download time was not the main concern.

However, in the last few years, technologies directly related to CCD performance have undergone significant developments. Taking into account only those which apply to full frame CCDs, i.e. the type used most in astronomy, we can consider the following advances:

- readout noise, which has been continuously dropping in all kind of cameras. Still on the edge of the professional market, the recently available L3Vision technology is able to offer cameras with sub-electron readout noise (e2v Technologies, 2002);

- multi output CCD, which increases the frame rate by dividing the data stream to be readout into several channels;
Table 3. Data transfer rate for different port architectures.

\begin{tabular}{lc}
\hline \hline Type & $\begin{array}{c}\text { Data transfer rate } \\
\left(\mathrm{Mbit} \mathrm{s}^{-1}\right)\end{array}$ \\
\hline Serial & 0.115 \\
Parallel Port EPP/ECP & $0.5-1$ \\
Firewire & 200 \\
USB 2.0 & 480 \\
Ethernet & $10 / 100 / 1000$ \\
\hline
\end{tabular}

- new data transfer interfaces, which have noticeably increased the throughput in comparison to parallel port (see Table 3). Apogee (2003), SBIG (2002) and Ethernaude (2001) constitute recent examples of this improvement, with the application of USB 2.0 and Ethernet interfaces to CCDs in the high-end amateur market. These kinds of initiative can supply readout rates typically 10 to 30 times faster than that offered by our port-parallel camera.

Therefore, the benefits to the fast drift scanning technique from all CCD improvements above are straightforward. On the one hand, lower readout noise will increase the SNR of the specklegrams. On the other hand, a faster readout rate will certainly decrease dead time and, as a result, low-level streaking between speckle frames would be effectively reduced.

\subsection{Limitations of the self-calibration technique}

The self-calibration method used here cannot be used in all situations. Indeed, the principal limitation is due to the zenith angle. As the zenith angle increases, the dispersion of the atmosphere elongates the speckles so that the speckle transfer function is no longer radially symmetric, and therefore the point source estimate generated is not an appropriate representation of the speckle transfer function at high zenith angles. This in turn can affect the relative astrometry and photometry derived from such data.

In considering differential photometry, one would expect that this is more sensitive to calibration effects than the astrometric results, since the process of deriving the magnitude difference amounts to estimating the fringe depth in the Fourier plane. If one uses a symmetric point spread function estimate to deconvolve an asymmetric binary power spectrum, the fringe depth can be severely affected while the fringe spacing and orientation would remain essentially the same.

It is also quite likely that in the case of a faint binary star, it is probably better to use a brighter binary to obtain the onedimensional trace simply due to signal-to-noise considerations.

\section{Summary and final remarks}

A new approach to performing CCD-based speckle imaging has been presented. Data obtained by those means have enough quality to give real scientific results, as shown for objects observed for this paper. 
In addition, a new approach for calibrating the power spectrum analysis has been introduced. It does not require point source observations, which gives a more effective use of observation time. Some limitations have been observed for this method for zenith angles above $30^{\circ}$ related to atmospheric dispersion. These conclusions can gain even more importance for the case of large telescopes. On the one hand, as they have the highest observing time pressure, self-calibration techniques would obviate point sources observations. On the other hand, if conveniently equipped with Risley prisms, they could be used to observe objects at low elevations without serious effects on the shape of speckles due to atmospheric dispersion. Thus, selfcalibration would presumably not be limited by elevation.

CCDs, far from being specialized detectors, are very common among instrumentation available in most astronomical observatories. The fast drift scanning enables low budget professional and high-end amateur observatories, which routinely use full-frame CCDs for stare imaging, to perform CCD speckle imaging as well. The performance of this technique will be significantly higher with new faster and less noisy cameras which are becoming available in the CCD market.

Acknowledgements. This work was supported in part by the DGICYT Ministerio de Ciencia y Tecnología (Spain) under grant No. AYA2001-3092. O. Fors was supported by a fellowship from DGESIC Ministerio de Educación, Cultura i Deportes (Spain), ref. AP97 38107939. Authors thank Observatorio Astronómico Nacional for facilities made available at Calar Alto. We would like to express our gratitude here to Christoph Flohr for making available his program SCAN.

\section{References}

Apogee Instruments Inc 2003, Alta Cameras Announcement (available at http://www.ccd.com/alta.html)

Baize, P. 1969, IAU Comm., 26 Inf., Circ. 48
Beletic, J. W., Zadnik, J. A., Tritsch, C. L., et al. 1991, BAAS, 24, 692

Docobo, J. A., \& Costa, J. M. 1990, PASP, 102, 1400

Douglass, G. G., Mason, B. D., Germain, M. E., et al. 1999, AJ, 118, 1395

e2v Technologies 2002, e2v Technologies L3Vision high sensivity imaging CCD for low light applications (available at http://e2vtechnologies.com/introduction/ prod_l3vision_nojs.htm)

Ethernaude Project 2001, Ethernaude Home Page (available at http://astrosurf.com/ethernaude)

Fors, O., Núñez, J., \& Richichi, A. 2001, A\&A, 378, 1100

Fors, O., Richichi, A., \& Núñez, J. 2003, A\&A, accepted

Fried, D. L. 1979, Optica Acta, 26, 597

Hartkopf, W. I., McAlister, H. A., \& Mason, B. D. 2002, Fourth Catalog of Interferometric Measurements of Binary Stars, United States Naval Observatory, Washington, USA (available at http://ad.usno.navy.mil/wds/int4.html)

Hartkopf, W. I., \& Mason, B. D. 2003, Sixth Catalog of Orbits of Visual Binary Stars, United States Naval Observatory, Washington, USA (available at http://ad.usno.navy.mil/wds/orb6.html)

Horch, E. P., Dinescu, D. I., Girard, T. M., et al. 1996, AJ, 111, 1681

Horch, E. P., Ninkov, Z., \& Slawson, R. W. 1997, AJ, 114, 2117

Horch, E., Ninkov, Z., van Altena, W. F., et al. 1999, AJ, 117, 548

Horch, E. P., Robinson, S. E., Meyer, R. D., et al. 2002, AJ, 123, 3442

Kluckers, V. A., Edmunds, M. G., Morris, R. H., et al. 1997, MNRAS, 284, 711

Korff, D. 1973, Opt. Soc. Am. J., 63, 971

Meng, J., Aitken, G. J. M., Hege, E. K., et al. 1990, Opt. Soc. Am. J., 7,1243

SBIG 2002, New USB Camera Announcement (available at http://www. sbig.com/sbwhtmls/newcameras.htm)

Scardia, M., Prieur, J. L., Koechlin, L., et al. 2002, IAU Comm., 26 Inf., Circ. 146

Starikova, G. A. 1982, SvAL 8, 166 (Pisma Astron. Zhur. 8, 306, 1982)

Zadnik, J. A. 1993, Ph.D. Thesis, Georgia Institute of Technology, Atlanta 